\title{
Clinical and Pathologic Complete Response to Gefitinib in a Patient with SqCLC Harboring EGFR p.E746_S752delinsV Mutation
}

\author{
Weitao Zhuang (D) $^{1,2}$ \\ Chao Zhang ${ }^{3}$ \\ Yong Tang (D) \\ Dan Tian' \\ Zihua Lan ${ }^{1,2}$ \\ Cheng Zeng' \\ Guibin Qiao (D)'
}

'Department of Thoracic Surgery, Guangdong Provincial People's Hospital, Guangdong Academy of Medical Sciences, Guangzhou, 510080, People's Republic of China; ${ }^{2}$ Shantou University Medical College, Shantou, 5I504I, People's Republic of China; ${ }^{3}$ Guangdong Lung Cancer Institute, Guangdong Provincial People's Hospital, Guangdong Academy of Medical Sciences, Guangzhou, 510080 , People's Republic of China
Correspondence: Guibin Qiao Department of Thoracic Surgery, Guangdong Provincial People's Hospital, Guangdong Academy of Medical Sciences, Guangzhou, 510080, People's Republic of China

Email guibinqiao@I26.com

\begin{abstract}
Development of targeted therapies for squamous cell lung cancer (SqCLC) is currently limited by the prevalence of activating mutations and their predicting power of treatment efficacy. In the present study, we describe a case of treatment-naïve stage IIIB SqCLC that harbored a rare epidermal growth factor receptor (EGFR) $p$. E746_S752delinsV mutation with clinical complete response to neoadjuvant gefitinib. Pathological complete response was confirmed after surgical resection. No disease recurrence was documented after 20-month follow-up. This report suggested that firstgeneration EGFR tyrosine kinase inhibitor (TKI) could be an option in neoadjuvant context for advanced SqCLC patients harboring EGFR p.E746_S752delinsV mutation and highlighted the clinical benefits of EGFR testing in SqCLC patients who are females and never/former light smokers.
\end{abstract}

Keywords: epidermal growth factor receptor, squamous cell lung cancer, tyrosine kinase inhibitor, next generation sequencing, EGFR p.E746_S752delinsV mutation, EGFR, SqCLC, TKI, NGS

\section{Introduction}

Unlike sensitive epidermal growth factor receptor (EGFR) mutations where various tyrosine kinase inhibitors (TKIs) exhibit remarkable response, rare EGFR subtypes might be neglected due to low prevalence and controversial efficacy for different TKIs. A previous study of 432 non-small cell lung cancer (NSCLC) patients found no significant differences in clinical outcomes after TKI treatment across 39 subtypes of EGFR exon 19 deletions. ${ }^{1}$ This result was challenged by a more well-designed study recently, in which patients with uncommon 19delins demonstrated significantly longer median progression free survival than those with common 19delins after treated by first-generation TKIs. ${ }^{2}$ Currently, efficacy of EGFR-TKIs for SqCLC remained controversial with most of the studies showing inferior response rate and prognostic outcome. One recent study reported a much worse prognosis (progression-free survival, 4.6 vs. 11.0 months, $\mathrm{p}<0.001)$ in EGFR-mutant SqCLC $(\mathrm{n}=28)$ than EGFRmutant adenocarcinoma $(n=41)$ after treating with EGFR-TKIs. ${ }^{3}$ There is even less knowledge to its efficacy in SqCLC harboring uncommon mutations. Herein, we present the clinical course of a patient with SqCLC bearing an EGFR p.E746_S752delinsV mutation who responded well to gefitinib as neoadjuvant monotherapy. 


\section{Case Presentation}

A 67-year-old Chinese female never-smoker presented to our clinic with persistent dry cough for 2 months. Her past medical history was noted for mild anemia and a 10-year history of hypertension, without any other documented health problem such as chronic respiratory disease or malignancy. Computed tomography (CT) revealed a $4.3 * 3.5 \mathrm{~cm}$ lung mass and a suspicious $7 \mathrm{~mm}$ solitary metastasis in the right lower lobe, as well as enlarged hilar and mediastinal lymph nodes (group 2R, 4R, 6, 7, 10R) (Figure 1A). She was then admitted for further investigation. Positron emission tomography (PET) scan was recommended but refused by patient due to its high cost. Alternatively, she was sent for cerebral magnetic resonance imaging and bone scan which did not suggest any metastatic sign. Biopsy of the lung mass confirmed a pure keratinizing squamous cell carcinoma which was negative for EML4-ALK fusion (Figure 1B). Next generation sequencing (NGS) targeting eight driver mutational genes (ALK, BRAF, EGFR, ERBB2, KRAS, MET, RET and ROS1) highlighted an EGFR p.E746_S752delinsV mutation (abundance 2.91\%). She was clinically diagnosed as stage IIIB (T3N2Mx) SqCLC. A multidisciplinary meeting was organized to discuss the treatment strategy for this patient. Considering that this was a never-smoker SqCLC bearing EGFR mutation, the patient was offered the first-generation EGFR TKI gefitinib (250 $\mathrm{mg}$ qd) as neoadjuvant regimen, and it was welltolerated with grade 1 abdominal pain, and grade 1 fatigue. The tumor rapidly shrank down (Figure 1C) and nearly disappeared after 11 weeks of treatment with normal serum level of tumor markers (Figure 1D). The short-axis diameter of the largest lymph node (4R) decreased from
$1.4 \mathrm{~cm}$ to $0.8 \mathrm{~cm}$. She continued with the same regimen for another 2 months and received right lower lobectomy and systemic lymphadenectomy via uniportal video-assisted thoracoscopic surgery. Pathological examination found no residual tumor cell in both primary lesion (Figure 1E) and draining lymph nodes (Figure 1F). This patient continued with a 5-month course of gefitinib postoperatively, and then self-withdrew due to a grade 3 abdominal pain, grade 2 fatigue, and grade 2 anorexia. She remains disease-free and is on active follow-up as of the writing of this report (Figure $1 \mathrm{G}$ and $\mathrm{H}$ ).

\section{Discussion}

SqCLC is typically associated with a history of heavy smoking, and is therefore a histological subtype with a high overall mutation burden but limited number of specific targetable sites. ${ }^{4}$ For this reason, patients with SqCLC do not benefit from major advances in the development of targeted therapeutics such as EGFR inhibitors which demonstrate exquisite activity in lung adenocarcinomas (LADC). It was reported that EGFR-TKIs only yielded a response rate of $22 \%$ to $38 \%$ in SqCLC among those with activating mutations, as opposed to a response rate of about $70 \%$ in LADC. 5,6 Given that the low prevalence of activating mutations and its poor predicting power for treatment efficacy of TKI in SqCLC, molecular testing for EGFR is not routinely recommended, except for those who are females or non-smokers. ${ }^{7,8}$

To the best of our knowledge, this report is the first to describe a treatment-naïve SqCLC which harbored EGFR p.E746_S752delinsV mutation with pathological complete response to gefitinib. Unlike LADC, the therapeutic

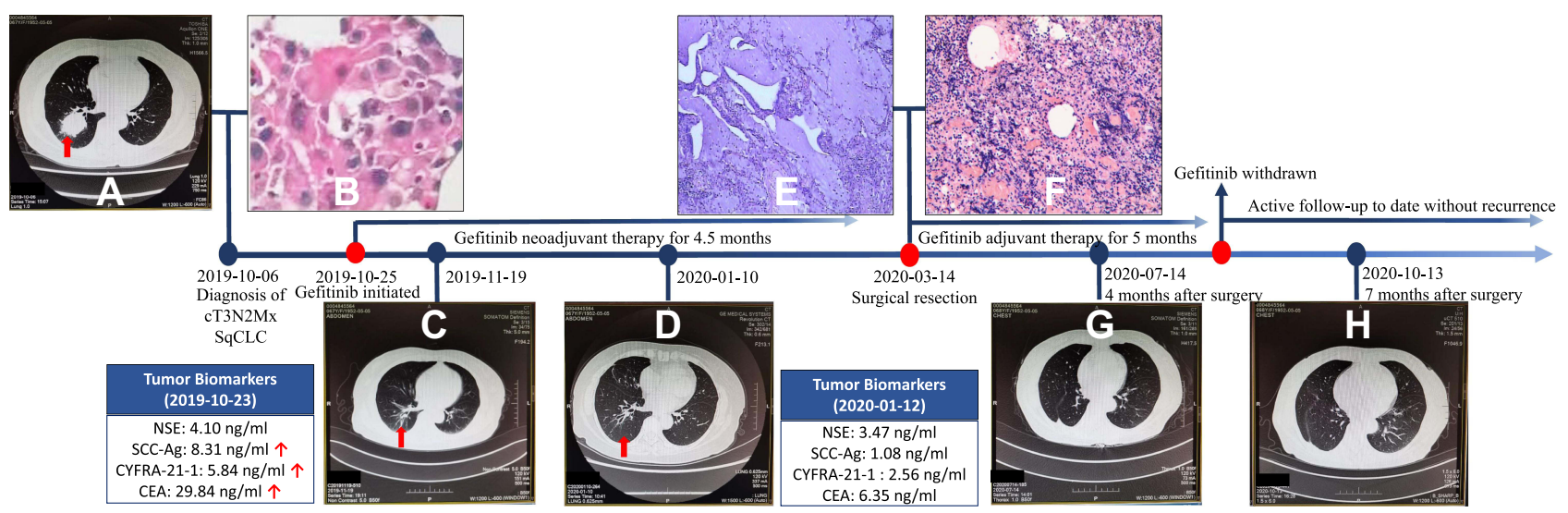

Figure I Timeline of the clinical course. Thoracic CT images of the tumor at baseline (A), after neoadjuvant treatment with gefitinib (C and $\mathbf{D})$, and at postoperative follow-up ( $\mathbf{G}$ and $\mathbf{H}$ ); Hematoxylin-Eosin stained slide of the core needle biopsy (B), resected primary lesion (E) and resected lymph node (F).

Abbreviations: NSE, neuron-specific enolase; SCC-Ag, squamous cell carcinoma antigen; CYFRA-2I-I, cytokeratin 19 fragment; CEA, carcinoembryonic antigen. 
repertoire for SqCLC is rather limited. As per the guidelines from National Comprehensive Cancer Network (NCCN) or Chinese Society of Clinical Oncology (CSCO), the stage IIIB NSCLC should be treated by definitive concurrent chemoradiation (Evidence level: category I) or by surgical resection with adjuvant chemotherapy (Evidence level: category IIA). ${ }^{8,9}$ The presence of EGFR mutation and administration of gefitinib offered a chance for our patient to achieve R0 resection and a better prognostic outcome. Nevertheless, the impact of neoadjuvant therapy with TKI in EGFR-mutated locally advanced lung cancer remains controversial. The most recent pooled analysis of 124 patients from five prospective clinical trials demonstrated a satisfactory surgical outcomes and good tolerability to use EGFR TKI in the neoadjuvant treatment of resectable NSCLC. ${ }^{10}$ However, results in terms of objective response rate (ORR) and survival are inconsistent in many studies. ${ }^{11}$ As the locally advanced NSCLCs are highly heterogeneous, a multidisciplinary team (MDT) involving oncologists, surgeons, pulmonologists and radiation oncologists is necessary to ensure a best practice of precision medicine. ${ }^{12}$ In the current report, MDT was a key step to decide the optimal therapeutic option, though limited evidence was available for clinical management of SqCLC with EGFR p.E746_S752delinsV mutation.

A previous study reported a 6-month progression free survival (PFS) on erlotinib in a patient with metastatic SqCLC harboring the identical EGFR mutation. ${ }^{13}$ Though not as good as the outcome in our study, the efficacy of EGFR TKIs was remarkable in both reports, which also underlined the prominent role of molecular features over histology in SqCLC. Interestingly, advanced SqCLC patients with other common subtypes of EGFR mutations demonstrated a shorter PFS ( $\mathrm{n}=22, \mathrm{PFS}=3.94$ months, 95\% CI, 2.73-5.15) on TKI therapy than these two patients with the rare EGFR p.E746_S752delinsV mutation. ${ }^{14}$ This phenomenon echo the result reported by Peng et al and merited further discussion in the future studies. ${ }^{2}$ Improta et al further divided patients with uncommon exon 19 EGFR mutations to frame-shift mutation and in-frame complex deletion, and found better response to TKI in the former subtype of mutation. They reported a 5-month PFS after targeting EGFR p.E746_S752delinsV with gefitinib in a patient with stage IV adenosquamous carcinoma, in contrast to a PFS of 18 to 48 months in patients harboring frame-shift mutations. ${ }^{15}$ Given the rarity of EGFR p. E746_S752delinsV mutation, future pooled analysis is warranted to validate the efficacy of EGFR TKI, especially in the context of neoadjuvant therapy.

Although resistance to gefitinib and subsequent tumor progression or recurrence was not observed in our case, resistance to afatinib has been noted in several previous reports after acquiring p.T790M mutation. ${ }^{13,16}$ Interestingly, EGFR p.G724S and p.C797S, the two emerging osimertinib-resistance mutations, seemed to exist exclusively in the context of specific 19Del mutants such as p.E746_S752delinsV, but not the most common 19Del variants. $^{16-18}$ In this context, rechallenge with gefitinib was found to have an outstanding response. ${ }^{16-18}$

There are several limitations to the current report. Firstly, the patient-reported outcome of health-related quality of life was not collected, which might lead to omission of some drug-related toxicities. Secondly, this patient is still under active follow-up, thus cancer recurrence could not be ruled out in the future. Thirdly, the clinical implication of this case-level report is limited by the unclear mechanism, which requires further validation by molecular experiments.

\section{Conclusion}

In conclusion, this report highlighted the clinical potentials of EGFR testing in SqCLC patients who are females and never/former light smokers, and also provided clinical evidence of first-generation EGFR-TKI in neoadjuvant context for advanced SqCLC patients harboring EGFR p. E746_S752delinsV mutation.

\section{Ethical Approval}

All procedures were in accordance with the ethical standards of the institutional and/or national research Committee(s) and with the 1964 Helsinki declaration and its later amendments. Institutional approval was waived to publish the case details.

\section{Patient Informed Consent}

Written informed consent was obtained from the patient for publication of this case report and any accompanying images in an anonymised manner.

\section{Acknowledgments}

This work was financially supported by the 2020 Guangdong Provincial Special Project for Popularization of Science and Technology Innovation (grant number: 2020A1414070007). 


\section{Disclosure}

The authors declare no conflicts of interest in this work.

\section{References}

1. Su J, Zhong W, Zhang X, et al. Molecular characteristics and clinical outcomes of EGFR exon 19 indel subtypes to EGFR TKIs in NSCLC patients. Oncotarget. 2017;8(67):111246-111257. doi:10.18632/ oncotarget. 22768

2. Peng $X$, Long $X$, Liu L, et al. Clinical impact of uncommon epidermal growth factor receptor exon 19 insertion-deletion variants on epidermal growth factor receptor-tyrosine kinase inhibitor efficacy in non-small-cell lung cancer. Eur $J$ Cancer. 2020;141:199-208. doi:10.1016/j.ejca.2020.10.005

3. Jin R, Peng L, Shou J, et al. EGFR-mutated squamous cell lung cancer and its association with outcomes. Front Oncol. 2021;14(11):680804. doi:10.3389/fonc. 2021.680804

4. Cancer Genome Atlas Research N. Comprehensive genomic characterization of squamous cell lung cancers. Nature. 2012;489 (7417):519-525. doi:10.1038/nature11404

5. Shukuya T, Takahashi T, Kaira R, et al. Efficacy of gefitinib for non-adenocarcinoma non-small-cell lung cancer patients harboring epidermal growth factor receptor mutations: a pooled analysis of published reports. Cancer Sci. 2011;102(5):1032-1037. doi:10.1111/ j.1349-7006.2011.01887.x

6. Wu JY, Wu SG, Yang CH, et al. Comparison of gefitinib and erlotinib in advanced NSCLC and the effect of EGFR mutations. Lung Cancer. 2011;72(2):205-212. doi:10.1016/j.lungcan.2010.08.013

7. Zhang Q, Zhu L, Zhang J. Epidermal growth factor receptor gene mutation status in pure squamous-cell lung cancer in Chinese patients. BMC Cancer. 2015;15(1):88. doi:10.1186/s12885-0151056-9

8. National Comprehensive Cancer Network. Non-small cell lung cancer (version 5). NCCN clinical practice guidelines in oncology; 2021. Available from: https://www.nccn.org/guidelines/guidelines-detail? category $=1 \& i d=1450$. Accessed September 6, 2021.
9. Chinese Society of Clinical Oncology. Guidelines of non-small cell lung cancer (2020). China: People's Medical Publishing House; 2020.

10. Sun L, Guo YJ, Song J, et al. Neoadjuvant EGFR-TKI therapy for EGFR-mutant NSCLC: a Systematic Review and Pooled Analysis of Five Prospective Clinical Trials. Front Oncol. 2021;12(10):586596. doi: 10.3389 /fonc. 2020.586596

11. Franco F, Provencio M. Neoadjuvant treatment with tyrosine kinase inhibitors in patients with resectable non-small cell lung cancer. J Thorac Dis. 2019;11(10):4092-4095. doi:10.21037/jtd.2019.09.68

12. Pezzuto A, Terzo F, Graziani ML, et al. Lung cancer requires multidisciplinary treatment to improve patient survival: a case report. Oncol Lett. 2017;14(3):3035-3038. doi:10.3892/ol.2017.6511

13. Cortiula F, De Maglio G, Cangi MG, et al. Third-generation tyrosine kinase inhibitor in the treatment of epidermal growth factor receptor mutated squamous cell lung cancer: a tailored therapy approach. Ann Transl Med. 2019;7(1):14. doi:10.21037/atm.2018.12.42

14. Xu J, Chu T, Jin B, et al. Epidermal growth factor receptor tyrosine kinase inhibitors in advanced squamous cell lung cancer. Clin Lung Cancer. 2016;17(4):309-314.15. doi:10.1016/j.cllc.2015.11.009

15. Improta G, Zupa A, Natalicchio MI, et al. Uncommon frame-shift exon 19 EGFR mutations are sensitive to EGFR tyrosine kinase inhibitors in non-small cell lung carcinoma. Med Oncol. 2018;35 (3):28. doi:10.1007/s12032-018-1078-7

16. Chic N, Mayo-de-las-casas C, Reguart N. Successful treatment with gefitinib in advanced non-small cell lung cancer after acquired resistance to osimertinib. $J$ Thorac Oncol. 2017;12(6):e78-e80. doi:10.1016/j.jtho.2017.02.014

17. Zheng Q, Huang Y, Zhao H, et al. EGFR mutation genotypes affect efficacy and resistance mechanisms of osimertinib in T790M-positive NSCLC patients. Transl Lung Cancer Res. 2020;9(3):471-483. doi: $10.21037 /$ tlcr.2020.03.35

18. Peled N, Roisman LC, Miron B, et al. Subclonal therapy by two EGFR TKIs guided by sequential plasma cell-free DNA in EGFR-mutated lung cancer. $J$ Thorac Oncol. 2017;12(7):e81-e84. doi:10.1016/j.jtho.2017.02.023
OncoTargets and Therapy

\section{Publish your work in this journal}

OncoTargets and Therapy is an international, peer-reviewed, open access journal focusing on the pathological basis of all cancers, potential targets for therapy and treatment protocols employed to improve the management of cancer patients. The journal also focuses on the impact of management programs and new therapeutic

\section{Dovepress}

agents and protocols on patient perspectives such as quality of life, adherence and satisfaction. The manuscript management system is completely online and includes a very quick and fair peer-review system, which is all easy to use. Visit http://www.dovepress.com/ testimonials.php to read real quotes from published authors. 